\title{
Analytical Solution to a Simplified Circulatory Model using Piecewise Linear Elastance Function
}

\author{
JJS Chen ${ }^{1}$, T Heldt ${ }^{2}$, GC Verghese ${ }^{1}$, RG Mark ${ }^{2}$ \\ ${ }^{1}$ Department of Electrical Engineering and Computer Science \\ ${ }^{2}$ Harvard University - MIT Division of Health Sciences and Technology, \\ Massachusetts Institute of Technology, USA
}

\begin{abstract}
Circuit-based lumped parameter representations of the hemodynamic system are commonly used in teaching and research to analyze the system-level behavior of the circulation. While efficient numerical methods exist to solve the governing differential equations numerically, we present an analytical solution of a simplified hemodynamic model that is based on a piecewise linear ventricular elastance function. It will be shown that the analytical solution is very close to the simulation output of the circuit analog representation, and that computational time can be reduced significantly. Furthermore, we apply parameter estimation techniques to the analytical solution to construct an estimator for the elastance parameters based on experimental hemodynamic waveforms.
\end{abstract}

\section{Introduction}

Presently, many computational models of cardiovascular function are becoming rapidly available as biomedical engineering researchers combine knowledge of the human circulatory system with the processing power of modern computers to construct simulations of the pulsatile pressure and flow waveforms. Lumped parameter representations of arteries and veins coupled to time-varying elastance models of the heart have long been used to simulate system level cardiovascular dynamics. Simulation of these models involves implementation of the governing equations and numerical integration. Analytical solutions of the governing equations, when available, have several advantages. First, they provide an explicit mathematical description of the model behavior without the need for a numerical solver to integrate the differential equations, thereby reducing computational time. Second, analytical solutions, being a function of the model parameters, can be used to construct estimators for the parameters based on experimental hemodynamic waveforms.

The goal of this paper is to derive an analytical solution to a simplified lumped parameter hemodynamic model based on a piecewise linear elastance function. A closed-form analytical solution is made possible by a piecewise linear approximation of the time-varying elastance function and by making reasonable assumptions, all of which simplify the numerical integration of the governing differential equations. It will be shown that the analytical solution allows for the representation of the full voltage waveforms by a set of discrete-time points per cycle, which we term the discrete-time analytical solution (DTAS). The voltage waveforms and the simulation time of the analytical solution will be compared to those of the circuit simulation of the pulsatile model. Lastly, we apply estimation techniques to the analytical solution to construct an estimator for the contractility ratio.

\section{Pulsatile model}

The pulsatile model, as shown in Figure 1, is a simplified version of a lumped parameter representation of a previously published closed loop hemodynamic model [1]. The system is made up of three segments: a

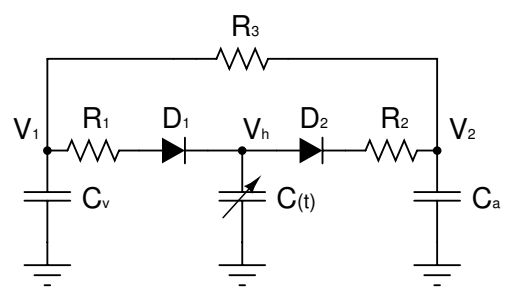

Figure 1. Pulsatile model.

venous, an arterial, and a ventricular compartment. The ohmic resistances $R_{1}, R_{2}$, and $R_{3}$ represent the resistance to venous return, the aortic outflow resistance, and the systemic arteriolar resistance, respectively. Ideal diodes $D_{1}$ and $D_{2}$, which mimic heart valves, ensure unidirectional flow through the system. The pumping action of the heart is represented using a time-varying ventricular compliance $C(t)$, where $C(t)$ is the inverse of elastance $E(t)$ (i.e. $\left.C(t)=\frac{1}{E(t)}\right)$. In close agreement with population- 
averaged experimental data [3] (shown in Figure 2), we define the time-varying elastance to be a piecewise linear function:

$$
E(t)=\left\{\begin{array}{cc}
\frac{E_{s}-E_{d}}{T_{s}} t+E_{d} & 0 \leq t \leq T_{s} \\
2 \cdot \frac{E_{d}-E_{s}}{T_{2}} t+3 E_{s}-2 E_{d} & T_{s}<t \leq \frac{3}{2} T_{s} \\
E_{d} & \frac{3}{2} T_{s}<t \leq T
\end{array}\right.
$$

$E_{s}$ and $E_{d}$ correspond to the end-systolic and diastolic elastances, respectively. $T_{s}$, the systolic time interval, is defined to be one-third of the cycle period $T$, i.e $T_{s}=\frac{1}{3} T$. The parameter values for the pulsatile model and elastance function are based on literature values [2] as indicated in Table 1.

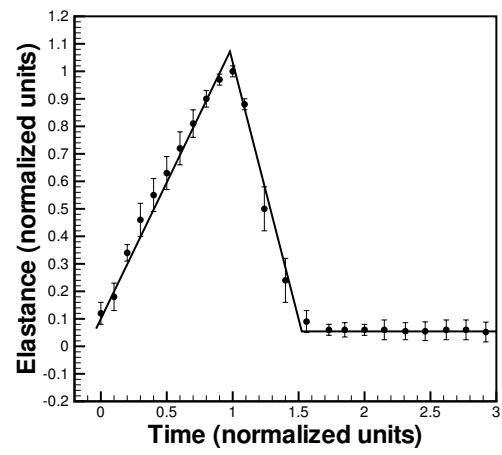

Figure 2. Piecewise linear function vs. experimental elastance. Data adapted from [3].

Table 1. Parameter assignments.

\begin{tabular}{cccccccc}
\hline$E_{s}$ & $E_{d}$ & $T$ & $C_{a}$ & $C_{v}$ & $R_{1}$ & $R_{2}$ & $R_{3}$ \\
\hline 2.5 & 0.1 & 1.0 & 2.0 & 100.0 & 0.03 & 0.01 & 1.0 \\
\hline
\end{tabular}

\section{Deriving the analytical solution}

In deriving the analytical solution to the pulsatile model, we solve the governing differential equations to obtain closed-form expressions for the voltage functions, making reasonable assumptions where possible/necessary. The onoff switching of the diodes $D_{1}$ and $D_{2}$, coupled with the piecewise linear properties of the elastance function $E(t)$, segment each cycle into seven distinct regions as shown in Figure 3. To facilitate the derivation of the analytical solution, the following notation is adopted for the $n$th region:

$\begin{array}{lcll}\text { start time } & t_{n-1} ; & \text { venous voltage } & V_{1 n}(t) \\ \text { end time } & t_{n} ; & \text { arterial voltage } & V_{2 n}(t) \\ \text { duration } & \bar{t}_{n} ; & \text { ventricular voltage } & V_{h n}(t) \\ \text { elastance } & E_{n}(t) ; & \text { charge on } C(t) & Q_{h n}(t)\end{array}$
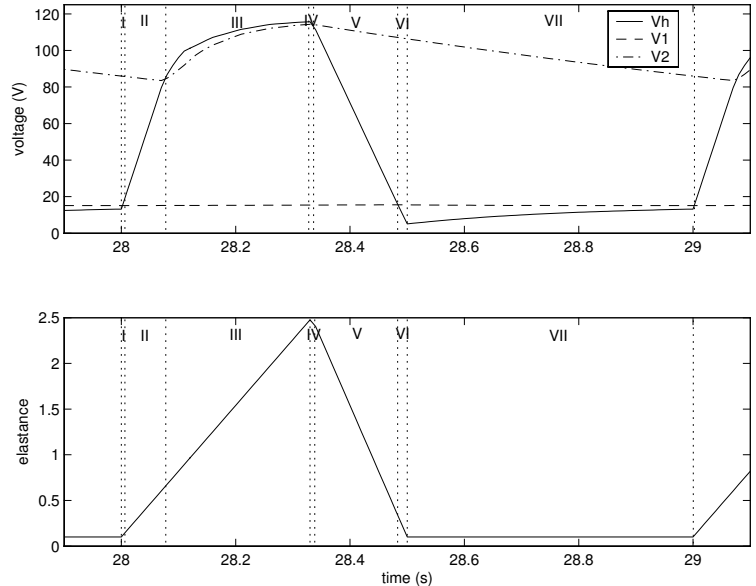

Figure 3. Voltage waveforms (top) and piecewise linear elastance function (bottom) divided into seven regions over one cycle.

Table 2. Definition of the regions of the cardiac cycle.

\begin{tabular}{cccc}
\hline Region & $D_{1}$ & $D_{2}$ & $E(t)$ \\
\hline I & on & off & linearly increasing \\
II & off & off & linearly increasing \\
III & off & on & linearly increasing \\
IV & off & on & linearly decreasing \\
V & off & off & linearly decreasing \\
VI & on & off & linearly decreasing \\
VII & on & off & constant \\
\hline
\end{tabular}

To derive the analytical solution for the $n$th region, first the initial conditions are obtained directly from the end of the preceding region. The equivalent circuit model and elastance function are defined for the region and the first-order governing differential equations is simplified through making reasonable approximations, in order to derive closed-form expressions for $V_{1 n}(t), V_{2 n}(t), V_{h n}(t)$ and $Q_{h n}(t)$. The time duration $\bar{t}_{n}$ is also computed to determine the end conditions of the region, which are used to initialize the succeeding region. Following is a demonstration of the approach outlined above using Region III as a concrete example. In this region, we assume $V_{1}$ to be constant over the cycle period, so $V_{1 n}(t)=V_{11}(0)$ for $n=$ $1, \ldots, 7 . R_{2}$ is assumed to be zero, as the potential difference between $V_{2}$ and $V_{h}$ is very small in the region. Note that the latter assumption does not allow for simulation of aortic stenosis. The equivalent circuit model is shown in Figure 4, where the capacitor $C_{v}$ is replaced by a DC voltage source. The initial conditions $E_{3}(0), V_{23}(0), V_{h 3}(0)$ and $Q_{h 3}(0)$ are determined directly from the end conditions of Region II. $E_{3}(t)$ is a linearly increasing function defined as, 


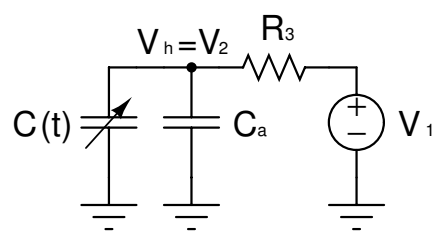

Figure 4. Equivalent circuit model for Region III

$$
E_{3}(t)=\frac{E_{s}-E_{d}}{T_{s}} t+E_{3}(0)
$$

A first-order governing differential equation in $Q_{h 3}(t)$ is obtained when we apply Kirchhoff's Current Law to the circuit model,

$$
\begin{gathered}
\dot{Q}_{h 3}(t)+\frac{V_{h 3}(t)-V_{13}(t)}{R_{3}}+C_{a} \dot{V}_{h 3}(t)=0 \\
\dot{Q}_{h 3}(t)=-\frac{E_{3}(t)+R_{3} C_{a} \dot{E}_{3}(t)}{R_{3}\left[1+C_{a} E_{3}(t)\right]} Q_{h 3}(t)+\frac{V_{11}(0)}{R_{3}\left[1+C_{a} E_{3}(t)\right]}
\end{gathered}
$$

where the second equaltiy follows from substituting $V_{h 3}(t)=Q_{h 3}(t) E_{3}(t)$ into Equation 3. The exact solution of the differential equation is, however, not in closed-form,

$$
Q_{h 3}(t)=\left(\frac{\beta t+\gamma}{\gamma}\right)^{\frac{1}{\beta C_{a} R_{3}}-1} e^{-\frac{t}{C_{a} R_{3}}}\left[K(t)+Q_{h 3}(0)\right]
$$

where

$$
\begin{gathered}
K(t)=\frac{V_{11}(0)}{R_{3}} \gamma^{\frac{1}{\beta C_{a} R_{3}}}-1 \int_{0}^{t} e^{\frac{\tau}{C_{a} R_{3}}}\left(\frac{1}{\beta \tau+\gamma}\right)^{\frac{1}{\beta C_{a} R_{3}}} \mathrm{~d} \tau \\
\beta=\frac{C_{a}}{T_{s}}\left(E_{s}-E_{d}\right) \\
\gamma=C_{a} E_{3}(0)+1
\end{gathered}
$$

The expression $e^{\frac{t}{C_{a} R_{3}}}\left(\frac{1}{\beta t+\gamma}\right)^{\frac{1}{\beta C_{a} R_{3}}}$ is approximately constant in the interval $\left[0, \bar{t}_{3}\right]$, where $\bar{t}_{3}=t_{3}-t_{2}$. From the definition of the region, $t_{3}=T_{s}$ and $t_{2}$ is obtained from Region II. A closed-form $Q_{h 3}(t)$ is obtained by replacing the expression with a constant $c$ where

$$
\begin{gathered}
c=\frac{a+b}{2} \\
a=\left[e^{\frac{\tau}{C_{a} R_{3}}}\left(\frac{1}{\beta \tau+\gamma}\right)^{\frac{1}{\beta C_{a} R_{3}}}\right]_{\tau=0} \\
b=\left[e^{\frac{\tau}{C_{a} R_{3}}}\left(\frac{1}{\beta \tau+\gamma}\right)^{\frac{1}{\beta C_{a} R_{3}}}\right]_{\tau=\bar{t}_{3}}
\end{gathered}
$$

Last, $V_{23}(t)=V_{h 3}(t)=Q_{h 3}(t) E_{3}(t)$, where the first equality follows from our assumption that $R_{2}=0$.
A method of setting up the initial conditions of the new cycle is required for the beat-to-beat propagation of the analytical solution. $V_{21 \text { new }}(0)$ and $V_{h 1 \text { new }}(0)$ are obtained directly from the end conditions of Region VII of the last cycle, while $V_{11 \text { new }}(0)$ is determined by applying the principle of charge conservation, which requires the total charge in the system at the start of the new cycle to equal that at the start of the preceding cycle.

The behavior of the voltage waveforms within each region is completely described by the analytical solution, so it suffices to represent the full waveforms by discrete-time points corresponding to each region's boundary conditions.

\section{Comparison of simulations}

In Table 3, we compare steady-state values of commonly used pressure numerics derived from the analytical solution (AS) and the circuit simulation (CS) of the pulsatile model. The maximum relative error between the two implementations across all numerics is $1.11 \%$. Figure 5

Table 3. Relative error of steady-state response.

\begin{tabular}{clccc}
\hline & & CS & AS & rel. error \\
\hline$V_{1}$ & mean & 15.25 & 15.08 & $1.11 \%$ \\
$V_{2}$ & mean & 99.72 & 100.62 & $0.90 \%$ \\
& systolic & 114.30 & 115.00 & $0.61 \%$ \\
& diastolic & 83.50 & 84.05 & $0.66 \%$ \\
$V_{h}$ & systolic & 115.75 & 115.00 & $0.65 \%$ \\
& end-diastolic & 13.18 & 13.15 & $0.23 \%$ \\
\hline
\end{tabular}

shows the response of the analytical solution (AS) and the circuit simulation (CS) of the pulsatile model to changes in the arteriolar resistance $R_{3}$. At time $t=30 \mathrm{~s}$, the resistance is stepped down from $1.0 \Omega$ to $0.5 \Omega$ and at time $t=60 \mathrm{~s}$, the process is reversed. Figure 6 compares the transient
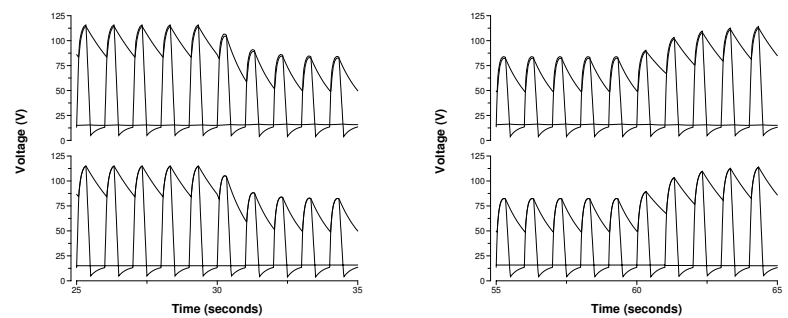

Figure 5. Transient response to changes in $R_{3}$. CS: upper panels; AS: lower panels

dynamics of the analytical solution and circuit simulation when the cycle period $T$ is stepped down from $1.0 \mathrm{~s}$ to $0.5 \mathrm{~s}$ at time $t=30 \mathrm{~s}$. The differences in the waveforms are hardly noticeable, and the maximum relative error for both transient simulations is no more than $1.79 \%$. 


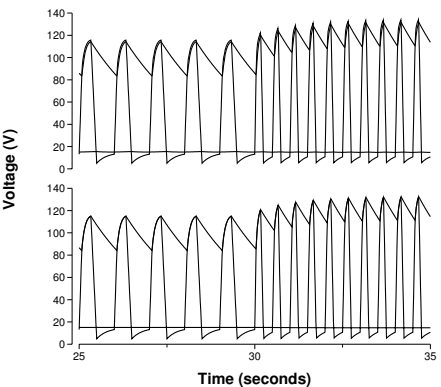

Figure 6. Transient response to changes in T. CS: upper panel; AS: lower panel

The analytical solution and the circuit simulation of the pulsatile model were implemented in the $\mathrm{C}$ programming language, where a standard fourth-order Runge-Kutta integrator was used to solve the differential equations numerically. The Gprof utility in LINUX was used to assess the CPU time of running each program for 10,000 cycles. Table 4 compares the averaged CPU times of the circuit simulation (CS), the discrete-time analytical solution (DTAS) and the full solution (AS) using different step sizes over 5 runs each.

Table 4. Comparison of CPU times

\begin{tabular}{cccccc}
\hline Variable & CS & \multicolumn{5}{c}{ AS } & DTAS \\
\hline step size (s) & $10^{-4}$ & $10^{-4}$ & $10^{-3}$ & $10^{-2}$ & - \\
CPU time (s) & 248.69 & 37.42 & 3.86 & 0.41 & 0.05 \\
\hline
\end{tabular}

\section{Parameter estimation}

The analytical solution is derived from the pulsatile model based on a time-varying ventricular elastance, and we reverse the process to construct an estimator for the elastance ratio, $E_{s} / E_{d}$, using $V_{h 2}(t)$.

$$
V_{h 2}(t) \approx \frac{V_{h 1}(0)}{T_{s}} \frac{E_{s}}{E_{d}} t+V_{h 1}(0)
$$

where we assume $E_{s} \gg E_{d}$ so that $\frac{E_{s}-E_{d}}{E_{d}} \approx \frac{E_{s}}{E_{d}}$. Equation 12 can be rewritten in matrix form i.e. $\mathbf{y}=\mathbf{H x}$ where

$\mathbf{y}=\left[\begin{array}{c}V_{h 2}[0] \\ \vdots \\ V_{h 2}[N]\end{array}\right] \mathbf{x}=\left[\begin{array}{c}\frac{V_{h 1}(0)}{T_{s}} \frac{E_{s}}{E_{d}} \\ \hline V_{h 1}(0)\end{array}\right] \mathbf{H}=\left[\begin{array}{c|c}0 & 1 \\ \Delta t & 1 \\ 2 \Delta t & 1 \\ \vdots & \vdots \\ N \Delta t & 1\end{array}\right]$

The maximum likelihood (ML) estimator for $\mathbf{x}$ is given by $\mathbf{x}_{\mathrm{ML}}=\left(\mathbf{H}^{\mathrm{T}} \mathbf{H}\right)^{-\mathbf{1}} \mathbf{H}^{\mathrm{T}} \mathbf{y}$. Assuming the terms are independent,

$$
\left[\frac{E_{s}}{E_{d}}\right]_{M L} \approx T_{s}\left[V_{h 1}(0)\right]_{M L}^{-1}\left[\frac{V_{h 1}(0)}{T_{s}} \frac{E_{s}}{E_{d}}\right]_{M L}
$$

where $T_{s}$ is easily computed from the cycle period $T$.

\section{Conclusions}

In this paper, we have derived an analytical solution to a simplified hemodynamic model based on a piecewise linear elastance function, that closely mimics clinical elastance data. We have shown that the steady-state and transient responses of the analytical solution are very close to the simulation output of the pulsatile model. It also allows for a discrete-time representation of the full voltage waveforms, which reduces the simulation time by a factor of 5000 . Furthermore, estimation of the contractility ratio based on hemodynamic waveforms is possible when we applied estimation techniques to the analytical solution.

In our main conclusion, the analytical solution is a highly efficient method to simulate hemodynamic waveforms, and can potentially aid in developing estimation methods for hemodynamic models. Future work will include a non-zero $R_{2}$, as well as addition of cardiovascular reflex mechanisms to allow for homeostatic control.

\section{Acknowledgments}

This work was partially supported by NASA through the NASA Cooperative Agreement NCC 9-58 with the National Space Biomedical Research Institute.

\section{References}

[1] Davis TL, Mark RG. Teaching physiology through simulation of hemodynamics. Computers in Cardiology 1990; 17: 64952.

[2] Heldt T, Shim EB, Kamm RD, Mark RG. Computational modeling of cardiovascular response to orthostatic stress. J. Appl. Physiol. 2002; 92:1239-54.

[3] Senzaki H, Chen CH, Kass DA. Single-beat estimation of end-sytolic pressure-volume relation in humans. Circulation. 1996;94:2497-506

Address for correspondence:

George Verghese, $\mathrm{PhD}$

Department of Electrical Engineering and Computer Science MIT Room 10-093 / Cambridge, MA 02139 / USA

verghese@mit.edu 